\title{
Stage IV Hodgkin's Disease presenting with spinal epidural involvement and cauda equina compression as the initial manifestation: Case report
}

\author{
Ahmet Toprak ${ }^{1}$, Nihat Kodalli ${ }^{2}$, Tulin Budak Alpdogan ${ }^{1}$, Adnan Giral ${ }^{1}$, Cigdem Ataizi Celikel $^{3}$, Nevzat Gurmen ${ }^{2}$ \\ and Mahmut Bayik ${ }^{1}$ \\ ${ }^{1}$ Department of Hematology and Immunology, Marmara University School of Medicine, Istanbul-Turkey; \\ ${ }^{2}$ Department of Radiology, Marmara University School of Medicine, Istanbul-Turkey; ${ }^{3}$ Department of Pathology, \\ Marmara University School of Medicine, Istanbul-Turkey
}

\begin{abstract}
Hodgkin's disease very rarely presents clinically, initially with a paraspinal mass, but this should be considered in the differential diagnosis. A patient presenting with back pain was diagnosed to have Stage IV Hodgkin's disease. MRI revealed an extradural and intraspinal soft tissue mass with bone infiltration. The importance of MRI in the early evaluation of a paraspinal mass and in determining the extent of the disease is emphasized.
\end{abstract}

Keywords: Hodgkin's Disease; spinal epidural involvement; cauda equina compression; MRI

\section{Introduction}

The frequency of spinal cord compression, either at presentation or during the course of Hodgkin's disease (HD), is 3.0 to $7.6 \%$ in different series. ${ }^{1,2}$ At autopsy, as might be expected, the incidence is higher than is evident clinically. Magnetic resonance imaging (MRI) improves the sensitivity of diagnosis of the epidural mass and accompanying bone marrow infiltration. A 20 -year old male presenting with back pain due to an epidural and an intraspinal mass was diagnosed as having Stage IV HD.

\section{Case report}

A 20-year-old male patient was admitted with a 5 months history of back pain referring to his left leg. Additionally, he had a 10 day history of fever and night sweats. The neurological examination was within normal limits. Hepatomegaly and splenomegaly were observed, but there was no peripheral lymphadenopathy.

Lumbar MRI examination revealed bone lesions within the sacrum and iliac wings with a high signal intensity on T2-weighted images indicating bone marrow infiltration. There was a lumbar epidural mass between levels L4 and S1. An additional intraspinal lesion was revealed with an extension through the spinal foramina towards spinal canal (Figure 1a and b). There were multiple lymphadenopathies with a maximal diameter of $2 \mathrm{~cm}$, visualized in the retrocaval, aortopulmonary and aortic arch regions on thoracic computer tomography (CT) scan. Additionally, there was subpleural parenchymal

Correspondence: N Kodalli, MD nodular infiltration in the lateral segment of the medial lobe of the right lung and lingular segment of the left lung and patchy infiltration in the lower lobes of both lungs. On abdominal CT, prominent hepatosplenomegaly and multiple retrocrural, mesenteric and aortocaval lymphadenopathies were detected. Nodular sclerosing HD was demonstrated by histopathological examination of bone marrow and left paratracheal lymph node (Figure $2 \mathrm{a}$ and $\mathrm{b}$ ).

The patient received standard alternate c-MOPPABVD (cyclophosphamide, vincristine, procarbazine, prednisone-doxorubicin, bleomycin, vinblastine, dacarbazine) protocol chemotherapy following the diagnosis. On repeated lumbar CT, regression of the paravertebral and intraspinal masses was observed following the first cycle of chemotherapy and further radiotherapy or surgical intervention to the spinal lesion was not considered (Figure 3b). Because of high risk of relapse in our patient, the standard alternate c-MOPP-ABVD chemotherapy protocol was completed in 12 months, although complete remission of the disease was confirmed with the CT scan, bone marrow aspiration and biopsy, and gallium scintigraphy at the sixth month of therapy. His disease is in complete remission without further treatment (Figure $4 \mathrm{a}$ and $\mathrm{b}$ ).

\section{Discussion}

Extranodal presentations account for 15 to $30 \%$ of all lymphoma cases. ${ }^{3}$ The propensity for non-Hodgkin's lymphoma (NHL) to involve and present in the extradural space is greater than that of HD (with a ratio of 3.27/1). ${ }^{4}$ The frequency of spinal cord compression in HD, either at presentation or during 
the course of disease varies between 3.0 to $7.6 \%$ in different series of HD. ${ }^{1,5}$ The incidence of spinal cord

a

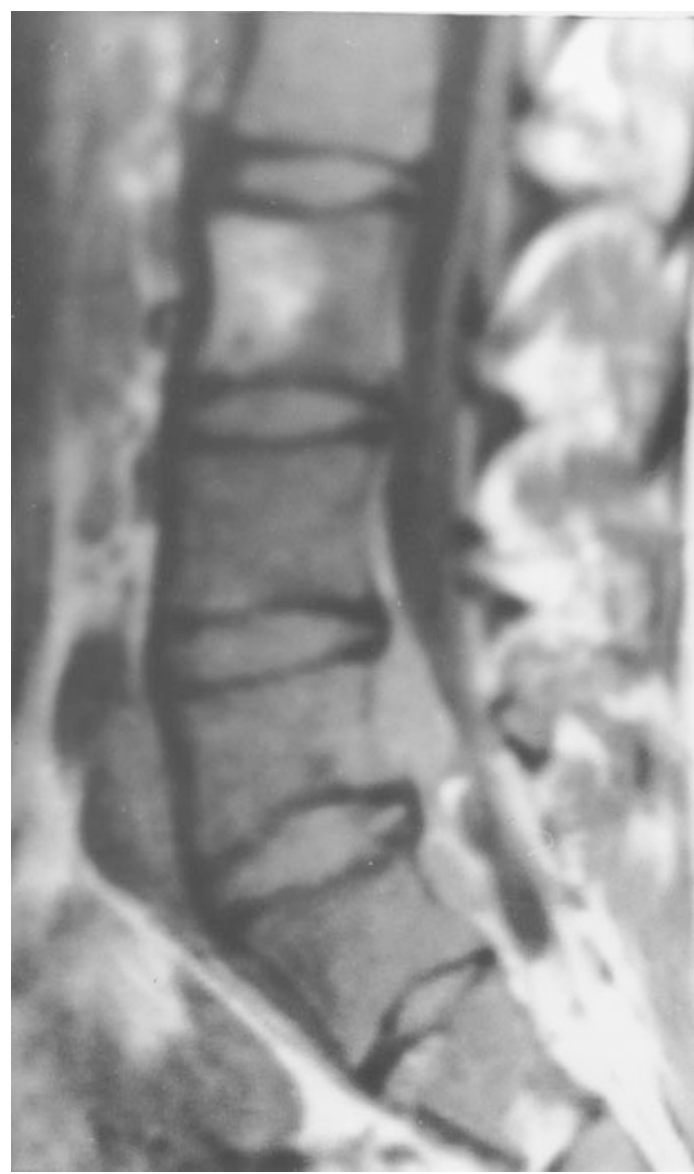

b

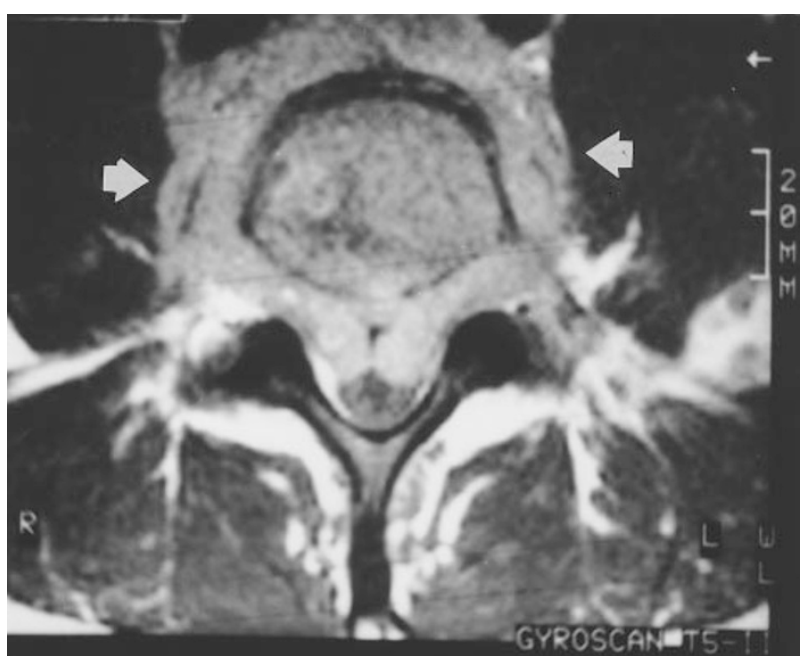

Figure 1 (a) T1-weighted sagittal spin-echo image shows epidural extension of the soft tissue lesion towards the spinal canal and compression of cauda equina. (b) Proton-density axial MRI (TR:1100, TE:40) shows the extension of the same lesion through the spinal foramina into spinal canal (arrow) and paravertebral space (arrow) compression at presentation $(0.8 \%)$ in $\mathrm{HD}$ is much lower than that described in newly diagnosed NHL patients, where cord compression without any preceding sign of disease accounts for $1.5-2.2 \%$ of cases. ${ }^{6,7}$ In the big series of Yale, consisting of more than 600 HD patients, only one instance of HD presenting with epidural disease and spinal cord compression as the only apparent site of initial clinical involvement was described. ${ }^{8}$

The regions most commonly affected by spinal HD involvement are the upper thoracic and lumbar spine. ${ }^{1}$ Tumor growth along the intervertebral foramina is believed to be the most common route of spread to the epidural space. ${ }^{5}$ Vertebral involvement and collapse may be directly responsible for spinal compression. Concomitant vertebral bone involvement has been observed in $32-42 \%$ of the cases. 1,4

None of the available data clearly defines the optimal treatment for HD presenting with spinal mass. Some authors advocate a multidisciplinary approach including local radiation therapy with or without surgical

a

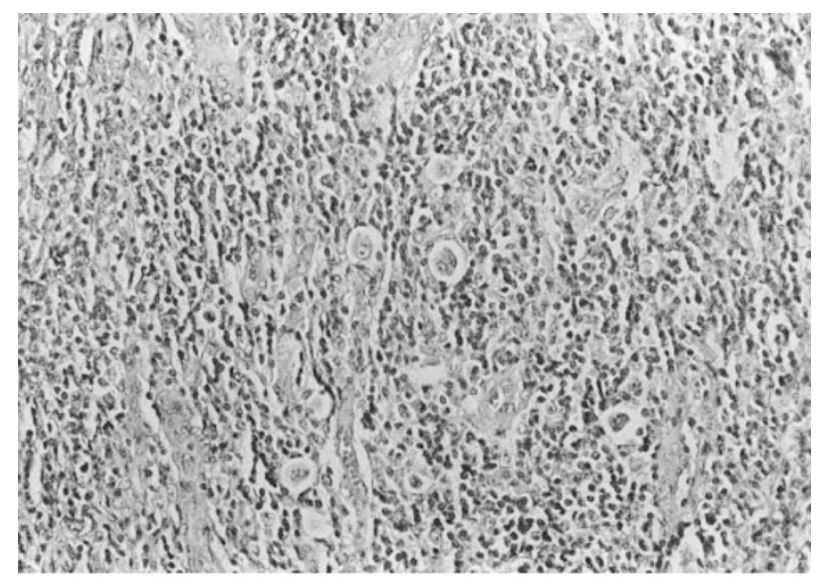

b

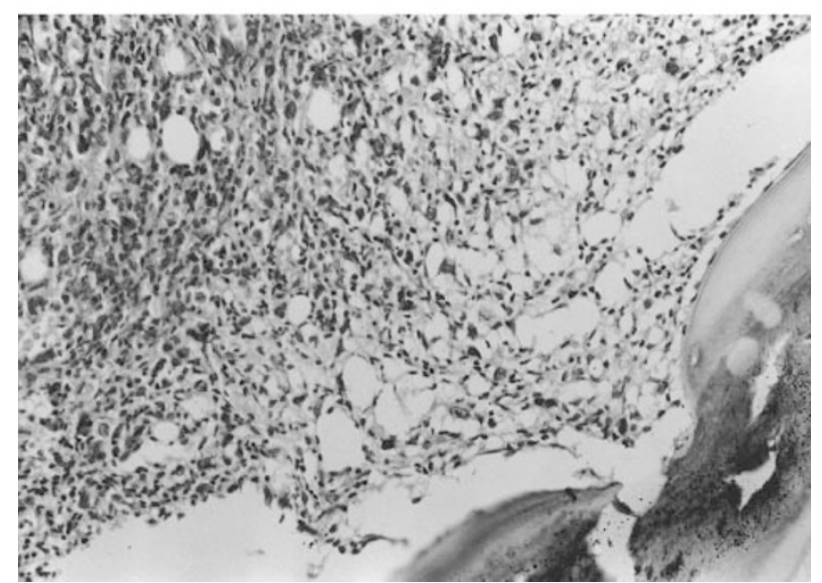

Figure 2 (a) Histologic section of a lymph node biopsy revealed Reed-Sternberg cells of lacunar type with a scant amount of fibrosis. (b) Histologic section of bone marrow discloses hypercellularity with dispersed lacunar cells and abundant eosinophilic leukocytes 
decompression, ${ }^{8}$ others favor chemotherapy as the primary treatment. ${ }^{9}$ The rate of complete remission in patients with advanced HD is $83 \%$ with MOPP-ABVD combination chemotherapy. ${ }^{10}$

Spinal foci of disease was found in $49 \%$ of 257 patients with HD and lymphosarcoma at autopsy, whereas radiologically demonstrable lesions were only present in $14.8 \%$ of the cases. ${ }^{2}$ MRI is a noninvasive procedure superior in the evaluation of the localization and the extent of a paraspinal soft tissue mass. Virtually the whole of the spinal canal is demonstrated. MRI is also helpful in the demonstration of bone marrow infiltration which is helpful in the staging of disease and can also be used as a guide for marrow biopsy. Early detection of spinal epidural lymphoma avoids serious complications of cord

a

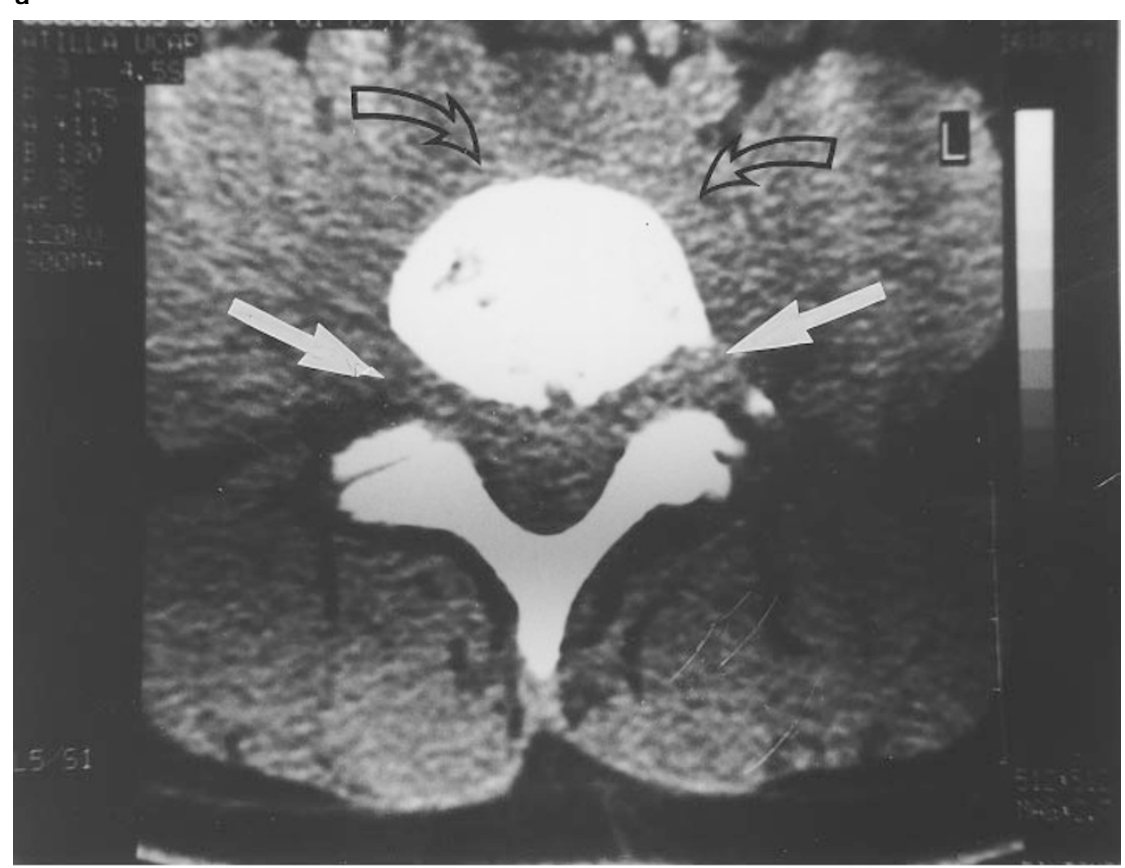

b

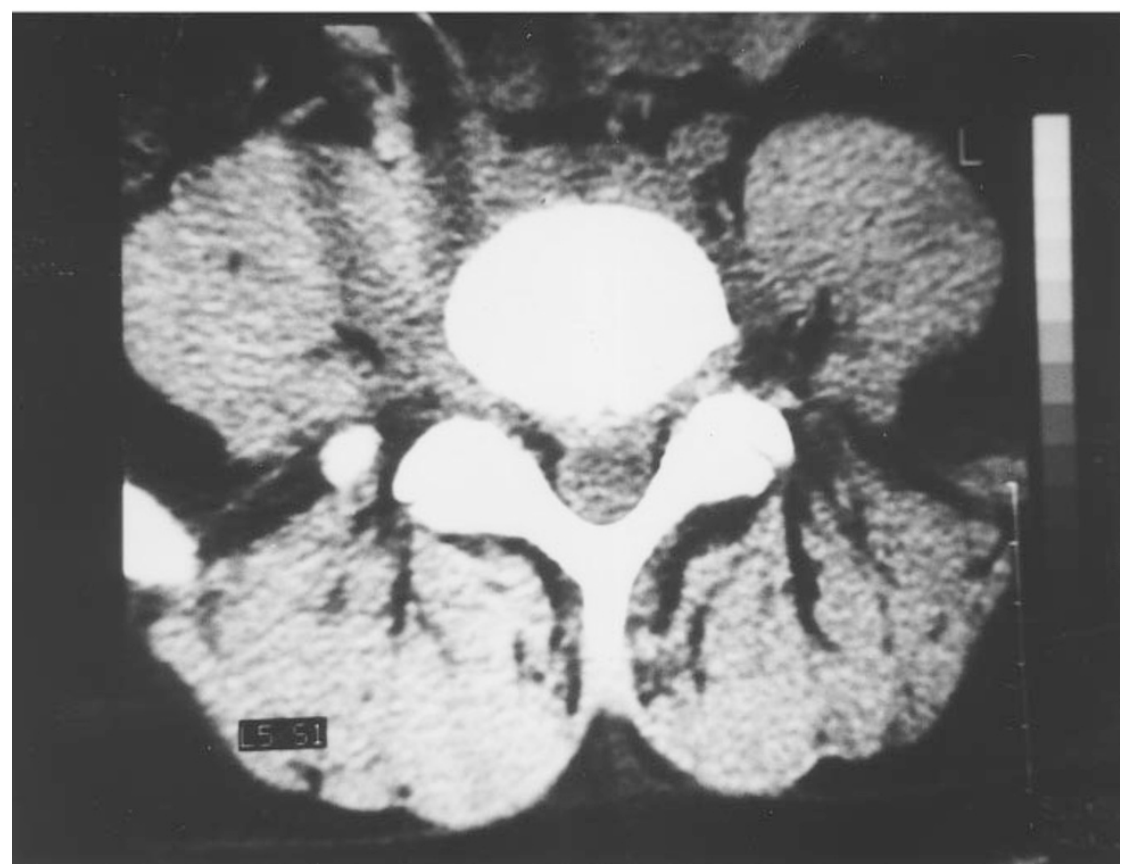

Figure 3 (a) CT scan without contrast at the level of L5/S1 shows intraspinal extension of the mass lesion through the foraminae (arrows) and paravertebral space (open arrows). (b) Complete regression of the mass lesion following chemotherapy is evident on CT scan at the level of L5/S1 after two months of chemotherapy 


\section{a}

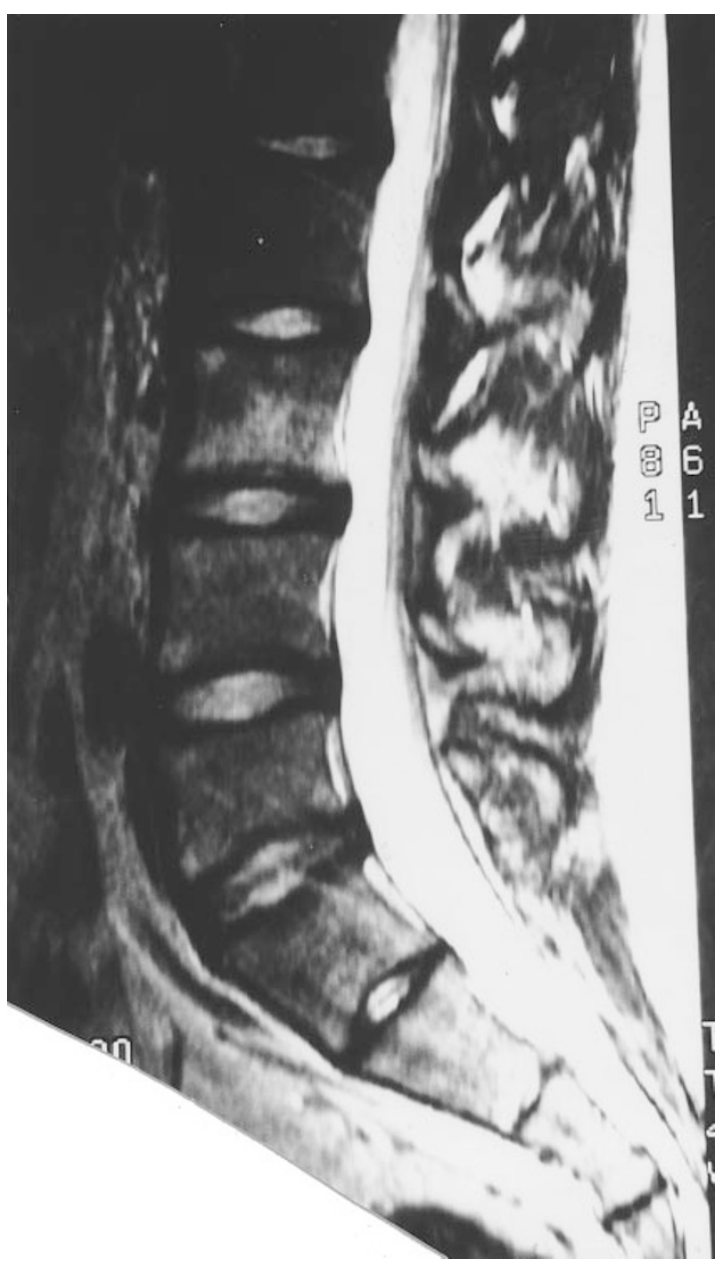

b

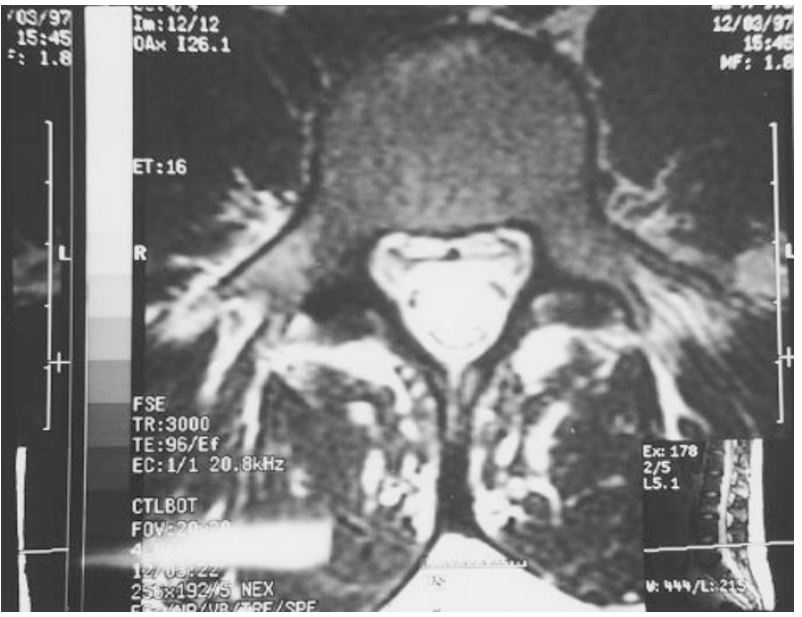

Figure 4 (a) T2-weighted sagittal spin echo image (TR:3000, TE:95) shows no evidence of the disease at 21st month of remission. (b) T2-weighted sagittal spin echo image at the level of L5/S1 (TR:3000, TE:96) shows no evidence of the disease at 21 st month of remission

compression such as paraplegia and sphincter impairment. We suggest that spinal MRI be performed without delay before the evolution of serious neurological deficits in patients with lymphoma and otherwise unexplained and significant back or radicular pain.

\section{References}

1 Friedman M, Kim TH, Panahon AM. Spinal cord compression in malignant lymphoma: treatment and results. Cancer 1976; 37: $1485-1491$

2 Vieta SO, Friedell HL. A survey of Hodgkin's disease and lymphosarcoma in bone. Radiol 1942; 39: 1-14.

3 Boddie AW Jr, Mullins JD. Extranodal lymphoma: Surgical and other therapeutic alternatives. Curr Probl Cancer 1982; 6: 4-64.
4 Haddad $\mathrm{P}$ et al. Lymphoma of the spinal extradural space. Cancer 1976; 38: $1862-1866$.

5 Mullins GM et al. Malignant lymphoma of the spinal epidural space. Ann Int Med 1971; 74: 416-423.

6 Epelbaum R et al. Non-Hodgkin's lymphoma presenting with spinal epidural involvement. Cancer 1986; 58: 2120-2124.

7 Lewitt LJ, Dawson DM, Rosenthal DS, Moloney WC. CNS involvement in the Non-Hodgkin's lymphoma. Cancer 1980; 45: $545-552$

8 Higgins SA, Peschel RE. Hodgkin's Disease with spinal cord compression. Cancer 1995; 75: 94-98.

9 Burch PA, Grossman SA. Treatment of epidural cord compression from Hodgkin's Disease with chemotherapy. Am J Med 1988; 84: $555-558$.

10 Canellos GP et al. Chemotherapy of advanced Hodgkin's disease with MOPP, ABVD, or MOPP alternating with ABVD. $N$ Eng $J$ Med 1992; 327: $1478-1484$. 